\title{
HEEGAARD DIAGRAMS OF LENS SPACES
}

\author{
R. P. OSBORNE
}

\begin{abstract}
Let $(M, F ; v, w)$ be a Heegaard diagram of $M$. The complexity of this diagram is the number of points in $v \cap m$. This is also the length of the relators in a group presentation naturally corresponding to this diagram. We give an example to show that a Heegaard diagram of minimal complexity need not have a cancelling pair of meridian disks. In terms of the presentation, this says that a minimal length presentation need not have a defining relator for one of the generators. This provides a counterexample to a conjecture of Waldhausen. Our example depends on the rather trivial observation that the shortest possible 2-generator presentation of the cyclic group of order 173 is $\left\langle a, b \mid a^{13} b^{2}, a^{-2} b^{13}\right\rangle$.
\end{abstract}

Let $M$ be a closed orientable 3-manifold. $M$ has a closed surface $F$ that separates it into two solid handlebodies of which the surface is the common boundary. This pair of handlebodies $(U, V)$ embedded in the manifold $M$ is called a Heegaard splitting $(M, F)$ of $M$. If one wants to describe a Heegaard splitting one needs to specify how the two handlebodies meet each other in $M$. This is done by giving a Heegaard diagram. To get a Heegaard diagram choose a complete set of meridian disks $u, v$ for the handlebodies $U$ and $V$; that is, $u$ is a set of disks that cuts the handlebody $U$ into a ball, similarly for $v$ in $V$. Now $(u \cup v) \cap F$ gives a system of simple closed curves on $F$ and the quadruple $(M, F ; u, v)$ is called a Heegaard diagram for $M$. By a slight adjustment one may assume that $u \cap v$ is a finite set of crossing points. The complexity of the Heegaard diagram is the number of points in $u \cap v$. Given a Heegaard diagram $(M, F ; u, v)$ one may increase the genus of the diagram in a rather trivial way. Simply drill an unknotted hole through one of the handlebodies and attach this hole to the complementary handlebody. This increases the genus of the Heegaard surface by one and is called a handle addition. A Heegaard diagram $(M, F ; u, v)$ has a cancelling pair of handles if there are disks $D_{1} \in u$ and $D_{2} \in v$ such that $\partial D_{1} \cap \partial D_{2}$ consists of a single (crossing) point (see also [W2]). Waldhausen [W1] conjectured that if a Heegaard diagram of minimal complexity is not a Heegaard splitting of minimal genus, then this diagram must contain a cancelling pair of handles. We give a Heegaard diagram for the lens space $L_{173,78}$ which has minimal complexity among genus 2 diagrams for $L_{173,78}$ but contains no cancelling pair of handles. To understand our example we need to consider group presentations that arise from Heegaard diagrams. Suppose $(M, F ; u, v)$ is a Heegaard diagram with $u=\left\{u_{1}, u_{2}, \ldots, u_{n}\right\}$ and $v=$ $\left\{v_{1}, v_{2}, \ldots, v_{n}\right\}$. Orient the elements $\partial u_{i}$ and $\partial v_{j}$ for each $i, j=1,2, \ldots, n$. Corresponding to each oriented simple closed curve $\partial v_{j}$ we get word in the free

Received by the editors March 9, 1981 and, in revised form, July 10, 1981.

1980 Mathematics Subject Classification. Primary 57M05; Secondary 20 F05. 
group $F\left(x_{1}, \ldots, x_{n}\right)$ as follows: Choose a starting point and travel around $\partial v_{i}$ following the orientation. Each time $\partial v_{j}$ intersects a point of $u_{i}$, we write $x_{i}$ if the crossing forms a right-handed pair of axes locally. Otherwise write $x_{i}^{-1}$. Proceeding around $\partial v_{j}$ gives a word in $F\left(x_{1}, \ldots, x_{n}\right)$ that describes how we cross each of the meridian disks in $u$. Let us call $R_{j}$ the word thus obtained. Thus, corresponding to each Heegaard diagram we have a presentation $\left\langle x_{1}, \ldots, x_{n} \mid R_{1}, \ldots, R_{n}\right\rangle$. A Heegaard diagram has a cancelling pair of handles if and only if the corresponding group presentation has a relator that defines a generator in terms of the other generators. The complexity of the diagram is just the length of the presentation.

THEOREM 1. For any choice of integers $m, n, p, q$ subject to the conditions $(m, p)=$ 1 and $(n, q)=1$, there is a Heegaard diagram for a lens space whose corresponding presentation is $\left\langle a, b \mid a^{m} b^{n}, a^{p} b^{q}\right\rangle$. Furthermore, if $m, n, p, q \neq 0$ and $\left.|m|+|p|\right\rangle 2$ and $|n|+|q|>2$ then the lens space is uniquely determined by this presentation.

Proof. This is the final result from [S].

THEOREM 2. The presentation $\left\langle a, b \mid a^{13} b^{2}, a^{-2} b^{13}\right\rangle$ is the shortest (in terms of total length of relators) two-generator, two-relator presentation of the cyclic group of order 173.

Proof. Suppose $\left\langle a, b \mid R_{1}, R_{2}\right\rangle$ presents the cyclic group of order 173. If we abelianize $R_{i}$ we get relations of the form $a^{m} b^{n}$ and $a^{p} b^{q}$. Now the group presented by $\left\langle a, b \mid a^{m} b^{n}, a^{p} b^{q}\right\rangle$ must also be of order 173. It follows that $|m q-n p|=173$. It is a routine matter to check that $m=13, n=2, p=-2$ and $q=13$ gives a presentation of minimal length. It follows that $\left\langle a, b \mid R_{1}, R_{2}\right\rangle$ has length at least as great as 30; if the length is 30 it cannot contain a defining relator.

From the above theorems we see that there is a Heegaard diagram of minimal complexity with presentation $\left\langle a, b \mid a^{13} b^{2}, a^{-2} b^{13}\right\rangle$ that contains no cancelling pair of handles. To show that this presentation determines $L_{173,78}$, one may use the decision process given in [S]. Of course, this lens space has a genus 1 Heegaard splitting.

The presentation $\left\langle a, b \mid a^{9} b^{2}, a^{-2} b^{9}\right\rangle$ corresponds to a minimal diagram of $L_{85,38}$, but the argument is a little more delicate than that for our first example because $\left\langle a, b \mid a^{12} b, a^{-1} b^{7}\right\rangle$ is a shorter presentation of the cyclic group or order 85 , but this presentation corresponds to $L_{85,7}$ as can be seen by the process of [S].

Birman and Montesinos [B\&M] have given an example to show that one of two conjectures of Waldhausen along these lines were incorrect, but were unable to decide which of these conjectures was incorrect. It is not difficult to write down the presentation corresponding to their genus 3 example and show that it is least a weak minimum (cf. [B\&M] for definition) but has no defining relator. This is surely an example of a diagram of minimal complexity not having a cancelling pair of handles, but actually proving this seems quite difficult because nothing is known about the group presented. Note: Birman and Montesinos showed that changing inner meridian disks gives a diagram with a cancelling pair of haildles but greater complexity. 
Generalizing our example for lens spaces to genus 3 and higher seems quite difficult because the shortest presentations do not correspond to Heegaard diagrams. For example, the cyclic group of order 173 can be presented by $\left\langle a, b, c \mid a^{4} c, a^{3} b^{5}, a^{-2} b c^{8}\right\rangle$. This presentation does not correspond to a Heegaard diagram of a lens space [O\&S].

The author wishes to thank Louise Moser for some very helpful conversations about this problem.

\section{REFERENCES}

[W1] F. Waldhausen, Some problems on 3-manifold, Proc. Sympos. Pure Math., vol. 32, Amer. Math. Soc., Providence, R. I., 1977; pp. 313-322.

[W2] __, Heegaard-Zerlegungen der 3-Sphäre, Topology 7 (1968), 195-203.

[B\&M] J. Birman and J. Montesinos, On minimal Heegaard splittings, Michigan Math. J. 27 (1980), 47-57.

[S] R. S. Stevens, Classification of 3-manifolds with certain spines, Trans. Amer. Math. Soc. 205 (1975), 151-166.

[O\&S] R. P. Osborne and R. S. Stevens, Group presentations corresponding to spines of 3-manifolds. I, Amer. J. Math. 96 (1974), 454-471.

Department of Mathematics, Colorado State Universtry, Fort Coluns, Colorado 80523 